\title{
Assessment of Factors that Influence Weaning from Long- term Mechanical Ventilation after Cardiac Surgery
}

\author{
Emília Nozawa, Eliane Kobayashi, Marta Erika Matsumoto, Maria Ignêz Zanetti Feltrim, \\ Maria José Carvalho Carmona, José Otávio Costa Auler Júnior
}

São Paulo, SP - Brazil

\begin{abstract}
Objective - To analyze parameters of respiratory system mechanics and oxygenation and cardiovascular alterations involved in weaning tracheostomized patients from longterm mechanical ventilation after cardiac surgery.
\end{abstract}

Methods - We studied 45 patients in their postoperative period of cardiac surgery, who required long-term mechanical ventilation for more than 10 days and had to undergo tracheostomy due to unsuccessful weaning from mechanical ventilation. The parameters of respiratory system mechanics, oxigenation and the following factors were analyzed: type of surgical procedure, presence of cardiac dysfunction, time of extracorporeal circulation, and presence of neurologic lesions.

Results - Of the 45 patients studied, successful weaning from mechanical ventilation was achieved in 22 patients, while the procedure was unsuccessful in 23 patients. No statistically significant difference was observed between the groups in regard to static pulmonary compliance $(p=0.23)$, airway resistance $(p=0.21)$, and the dead spacel tidal volume ratio $(p=0.54)$. No difference was also observed in regard to the variables $\mathrm{PaO}_{2} / \mathrm{FiO}_{2}$ ratio $(p=0.86)$, rapid and superficial respiration index $(p=0.48)$, and carbon dioxide arterial pressure $(p=0.86)$. Cardiac dysfunction and time of extracorporeal circulation showed a significant difference.

Conclusion - Data on respiratory system mechanics and oxygenation were not parameters for assessing the success or failure. Cardiac dysfunction and time of cardiopulmonary bypass, however, significantly interfered with the success in weaning patients from mechanical ventilation.

Key words: cardiac surgery, weaning, long-term mechanical ventilation

Instituto do Coração of the Hospital das Clínicas - FMUSP.

Mailing address: Emília Nozawa - Rua Mateus Grou, 539/71 - 05430-015 - São Paulo, SP, Brazil.

English version by Stela Maris C. e Gandour
The parameters of respiratory system mechanics and oxygenation are commonly used to wean patients from long-term mechanical ventilation ${ }^{1-4}$. Factors, such as advanced age, female sex, time of extracorporeal circulation, cardiac dysfunction, and low cardiac output, may lead patients to long-term mechanical ventilation ${ }^{5-8}$. However, few reports exist about the conjunct use of parameters of respiratory system mechanics and oxygenation, and cardiovascular alterations as factors involved in the successful weaning of patients from long-term mechanical ventilation after cardiac surgery.

In the postoperative period of cardiac surgery, patients are usually extubated as soon as the anesthetic effect ceases, and the ventilatory modality has little impact on the decision to extubate ${ }^{9}$. Approximately 3 to $6 \%$ of the patients, however, may require long-term mechanical ventilation due to the complexity of cardiac and pulmonary diseases or due to other systemic problems ${ }^{10}$. In these cases, the usual criteria for extubation, such as analysis of the arterial gases and determination of vital capacity and minute volume, many times fail to foretell the success of extubation ${ }^{11}$. The amount of material and financial resources consumed by these patients on long-term mechanical ventilation are high, and some studies were conducted in an attempt to find ways to reduce the costs ${ }^{5}$.

Our study aimed at analyzing the parameters of respiratory system mechanics and oxygenation, and cardiovascular alterations, and at assessing the factors involved in the success or failure of weaning tracheostomized patients from prolonged mechanical ventilation after cardiac surgery.

\section{Methods}

We studied 45 patients on long-term mechanical ventilation after cardiac surgery admitted to the Surgical Intensive Care Unit of the Instituto do Coração (InCor) of the Hospital das Clínicas of the Medical School of the University of São Paulo in the period from August 1997 to February 
1999. Thirty-four were males and 11 were females. All patients included in the protocol had undergone cardiac surgery with extracorporeal circulation and remained on long-term mechanical ventilation for more than 10 days after failure to wean, being then tracheostomized. Patients diagnosed with sepsis or mediastinitis, or with a temperature above $38^{\circ} \mathrm{C}$ or below $35^{\circ} \mathrm{C}$ were excluded from the study.

After tracheostomy, the patients were sedated with intravenous midazolam, and muscular relaxation was induced with pancuronium bromide for measurements of respiratory system mechanics. The Veolar-Hamilton ventilator (Switzerland) was used adjusted to the parameters of ventilatory modality and controlled volume to assure tidal volume of $8 \mathrm{~mL} / \mathrm{kg}$, inspiratory flow wave of the square type, and inspiratory pause of 0.5 seconds. The inspired fraction of oxygen and the positive end-expiratory pressure were adjusted to maintain a peripheral oxygen saturation $\geq 95 \%$ until the lower limit of oxygen concentration of $40 \%$ and positive end-expiratory pressure of $5 \mathrm{cmH}_{2} \mathrm{O}$ were achieved.

After adjusting the mechanical ventilator, the following measurements were taken directly from the panel of the respirator: tidal volume, peak inspiratory pressure, minute volume, respiratory rate, plateau inspiratory pressure, positive end-expiratory pressure, and inspiratory flow.

To assess respiratory system mechanics, the humidifier was removed from the circuit of the respirator ${ }^{12}$. The following measurements were taken: static compliance $\left(\mathrm{mL} / \mathrm{cmH}_{2} \mathrm{O}\right)$, airway resistance $\left(\mathrm{cmH}_{2} \mathrm{O} / \mathrm{L} / \mathrm{m}\right)$, dead space/ tidal volume ratio, rapid and superficial respiration index, $\mathrm{PaO}_{2} / \mathrm{FiO}_{2}$ ratio, and $\mathrm{PaCO}_{2}$.

Total static compliance was obtained dividing the tidal volume by the plateau pressure subtracted from the value of the positive end-expiratory pressure. Total airway resistance was calculated dividing the difference between the peak inspiratory pressure and the plateau pressure by the inspiratory flow. The dead space/tidal volume ratio (Cosmo Plus-9, Dixtal, São Paulo, Brazil) was obtained performing arterial blood gas analysis with $100 \%$ oxygen concentration. After spontaneous decurarization, and when the patient's clinical conditions allowed, the endotracheal tube was disconnected from the respirator and the rapid and superficial respiration index was measured at the first and fifth minutes. The parameters of respiratory system mechanics and oxygenation obtained were compared with the normal values ${ }^{13}$.

The type of surgical procedure, time of extracorporeal circulation, presence of myocardial dysfunction, and neurological alterations were analyzed. The time of extracorporeal circulation was considered longer or shorter than 120 minutes. Cardiac dysfunction was defined as cardiac index $<1.8 \mathrm{~L} / \mathrm{min} / \mathrm{m}^{2}$ in patients taking inotropic drugs or with ejection fraction $<50 \%$, or both, and was analyzed on echocardiography. Neurological alterations were the lesions clinically assessed and confirmed on cerebral computed tomography, excluding progressive neurological lesions.
Electrolytic disorders were promptly corrected and nutrition was performed via enteral or parenteral route, according to clinical conditions and nutritional assessment.

All patients underwent the weaning protocol standardized for the surgical ICU of InCor. The parameters used to assess weaning from the ventilator were as follows: hemodynamic stability; respiratory rate $<25 \mathrm{rpm} ; \mathrm{PaO}_{2} / \mathrm{FiO}_{2}>200$; carbon dioxide arterial pressure between 35 and $45 \mathrm{~mm} \mathrm{Hg}$; maximum inspiratory pressure $>25 \mathrm{cmH}_{2} \mathrm{O}$; tidal volume $>5$ $\mathrm{mL} / \mathrm{kg}$; and $\mathrm{SaO}_{2}$ maintained $>93 \%$. Weaning consisted of gradually reducing the ventilator parameters, such as respiratory rate (f), pressure supported ventilation (PSV), positive end-expiratory pressure, and fraction of inspired oxygen $\left(\mathrm{FiO}_{2}\right)$, until minimum parameters were reached, ie, $\mathrm{f}$ $=2 \mathrm{rpm}, \mathrm{PSV}=10 \mathrm{cmH}_{2} \mathrm{O}$, positive end-expiratory pressure $=$ $5 \mathrm{cmH}_{2} \mathrm{O}$, and $\mathrm{FiO}_{2}=0.4$. At that moment, gradual withdrawal from the ventilator was initiated under continuous nebulization with oxygen support and maintenance of oxygen saturation $>93 \%$ for 30 minutes in the morning and 30 minutes in the afternoon. In the nocturnal period, mechanical ventilation was maintained. Those periods of time were gradually increased until complete weaning from the ventilator was obtained, as shown in the weaning protocol in table I.

Success in mechanical ventilation weaning was defined as the patient's complete independence of the ventilator for a period $>48$ hours, maintaining oxygen saturation above $93 \%$ with oxygen support and no fatigue in the respiratory pattern (tachypnea, use of the accessory muscles, paradoxical movements, or asynchrony between the chest and the abdomen). Failure in mechanical ventilation weaning was defined as the patient's dependence on the ventilator for a period longer than 8 weeks (56 days) or when death occurred.

The study was approved by the scientific committee of InCor and the committee on ethics in research of the Hospital das Clínicas of the Medical School of the University of São Paulo.

All variables are described as mean and standard deviation. Data on success and failure were analyzed with the Student $t$ test, and the variables time of extracorporeal circulation and cardiac dysfunction were analyzed with the Fisher exact test. The statistical significance level adopted was $\mathrm{p}<0.05$.

\begin{tabular}{|lccc|}
\hline \multicolumn{4}{|c|}{ Table I - Weaning protocol } \\
\hline Protocol & \multicolumn{3}{c|}{$\begin{array}{c}\text { Time of nebulization } \\
\text { Period }\end{array}$} \\
\hline \multicolumn{4}{|c|}{ Afternoon } \\
\hline \\
\hline
\end{tabular}




\section{Results}

Of the 45 patients studied, 22 achieved independence from mechanical ventilation within 8 weeks and were discharged from the ICU. The other 23 patients did not succeed in being weaned from the mechanical ventilator. Of these 23 patients, 8 remained under ventilatory assistance for a period longer than 8 weeks, and 15 died. The patients' characteristics are shown in table II, and the description of the surgical procedures are shown in table III. Of the 5 patients who entered the protocol in the valvular group, 4 underwent surgical replacement of the aortic valve and 1 underwent surgical replacement of the mitral valve.

The variables respiratory system mechanics and oxygenation (static pulmonary compliance, airway resistance, dead space/tidal volume ratio, rapid and superficial respiration index, $\mathrm{PaO}_{2} / \mathrm{FiO}_{2}$ ratio, and carbon dioxide arterial pressure), when compared between the "successful" and "unsuccessful" groups, showed no statistically significant difference (tab. IV).

Of the patients studied, 27 (60\%) had cardiac dysfunction, $9(33 \%)$ of whom evolved to successful weaning, and $18(67 \%)$ evolved to unsuccessful weaning. Of the $18(40 \%)$

\begin{tabular}{|lccc|}
\hline \multicolumn{4}{|c|}{ Table II - Demographic characteristics of the patients } \\
\hline & Success $(\mathrm{n}=22)$ & Lack of success $(\mathrm{n}=23)$ & $\mathrm{P}$ \\
\hline & $63.3 \pm 12.9$ & $62.9 \pm 13$ & 0.87 \\
Age (years) & $73.2 \pm 12.9$ & $72.1 \pm 12.8$ & 0.77 \\
Weight (kilograms) & $27.5 \pm 6.3$ & $26.4 \pm 3.8$ & 0.50 \\
BMI $\left(\mathrm{kg} / \mathrm{m}^{2}\right)$ & $1.66 \pm 0.08$ & $1.62 \pm 0.13$ & 0.30 \\
Height $(\mathrm{m})$ & \\
\hline
\end{tabular}

\begin{tabular}{|c|c|c|}
\hline \multicolumn{3}{|c|}{ Table III - Types of surgical procedure in the 45 patients } \\
\hline Surgical procedure & Success & Lack of success \\
\hline Myocardial revascularization & 16 & 5 \\
\hline Associated procedure & 2 & 9 \\
\hline Valvular replacement & 1 & 4 \\
\hline Correction of AoA & 2 & 4 \\
\hline LV aneurysmectomy & 1 & 1 \\
\hline $\begin{array}{l}\text { Associated procedure }=\text { myoca } \\
\text { procedure; AoA }- \text { aneurysm of }\end{array}$ & $\begin{array}{l}\text { scularizati } \\
\text { ding aorta }\end{array}$ & $\begin{array}{l}\text { another associated } \\
\text { - left ventricle. }\end{array}$ \\
\hline
\end{tabular}

patients who had no cardiac dysfunction, $13(72 \%)$ evolved to independence from mechanical ventilation, and $5(28 \%)$ failed to wean from the mechanical ventilator. This difference was statistically significant ( $\mathrm{p}=0.016$ ) (fig. 1 ).

The time of extracorporeal circulation was shorter than 120 minutes in 23 patients, 15 (65\%) of whom evolved to success, and $8(35 \%)$ evolved to failure to wean from the mechanical ventilator. Of the 22 patients with extracorporeal circulation time longer than 120 minutes, $7(32 \%)$ evolved to success, and $15(68 \%)$ to failure to wean from mechanical ventilation, a statistically significant difference $(\mathrm{p}=0.038)$ (fig. 2).

In the group studied, 15 (33\%) of the 45 patients had neurological complications, 9 of whom succeeded in being weaned from the mechanical ventilator, while 6 did not.

\section{Discussion}

Our data show that $46 \%$ of the patients under mechanical ventilation were successfully weaned from ventilatory support. This percentage is compatible with those of other reports in the literature ${ }^{1,14,15}$. The causes of failure to wean from mechanical ventilation in these patients related mainly to the presence of cardiac dysfunction and prolonged time of extracorporeal circulation.

Several studies ${ }^{2,13}$ have recommended the use of static pulmonary compliance, an easily measured noninvasive variable, which proved to be a useful criterion to foretell success in weaning from mechanical ventilation in patients with pulmonary disorders. However, in our study, although static pulmonary compliance showed values below normal, it was not sensitive enough to differentiate the evolution of the patients in regard to weaning from mechanical ventilation. The reduction in static pulmonary compliance may have been due to procedures involved in surgery, such as sternotomy, extracorporeal circulation, surgical manipulation, and presence of drains ${ }^{16,17}$.

In our study, airway resistance was increased in all patients, but no significant difference was observed between the patients who evolved to independence from mechanical ventilation and those who evolved to unsuccessful weaning. Zanotti et $\mathrm{al}^{2}$, analyzing airway resistance in patients with chronic obstructive pulmonary disease

\begin{tabular}{|l|c|c|}
\hline \multicolumn{4}{|c|}{ Table IV - Comparison of the parameters of respiratory system mechanics and oxygenation between the groups that succeeded or failed to } \\
wean from prolonged mechanical ventilation \\
\hline
\end{tabular}




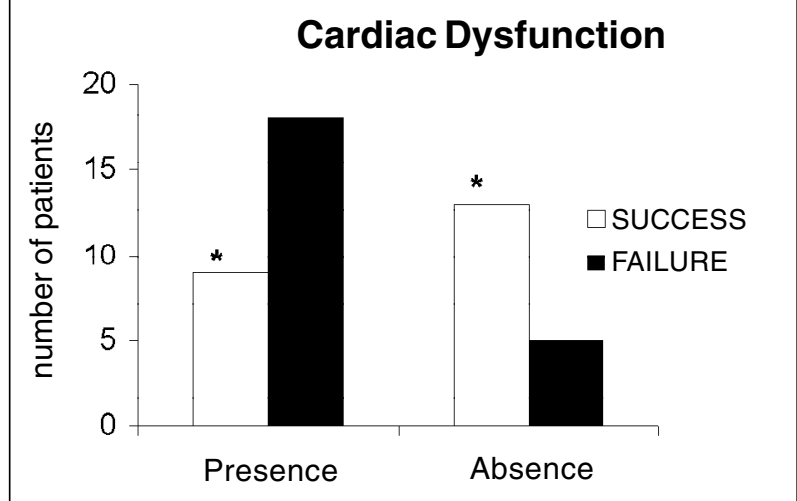

Fig. 1 - Cardiac dysfunction in the groups that succeeded or failed to wean from prolonged mechanical ventilation; $* \mathrm{p}<0.05$ for the comparison between those groups.

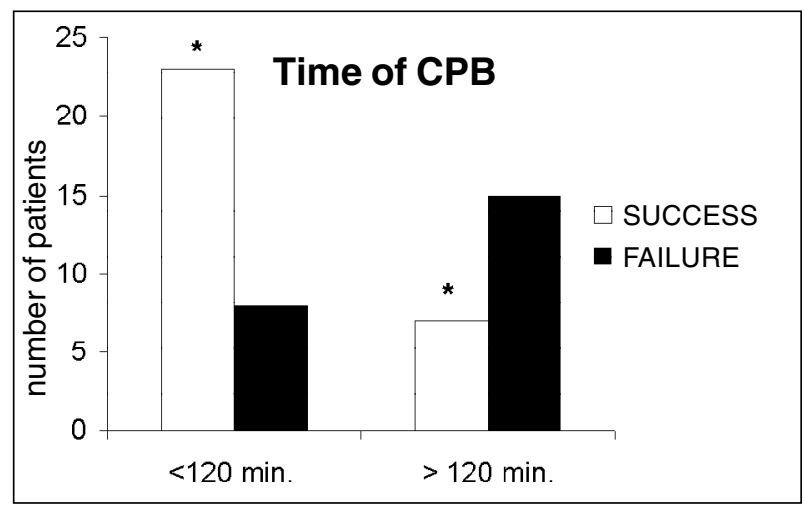

Fig. 2 - Time of extracorporeal circulation (CPB) in the groups that succeeded or failed to wean from prolonged mechanical ventilation; $* \mathrm{p}<0.05$ for the comparison between those groups.

(COPD), found similar results. On the other hand, Tobin ${ }^{18}$, in a recent study, reported that the increase in airway resistance in patients with COPD makes mechanical ventilation weaning difficult. The increase in airway resistance observed in our patients may have been due to edema of the airway walls, presence of fluids or secretions within the airway lumen, and a reduction in pulmonary functional volume ${ }^{16}$.

The dead space/tidal volume ratio has been reported to be predictive in mechanical ventilation weaning when associated with a system of scoring, including data on respiratory system mechanics and oxygenation ${ }^{1}$. Our study revealed that that variable was not significant in foretelling success in mechanical ventilation weaning, because both groups had values similar or close to normal.

The rapid and superficial respiration index variable is widely used to predict the results of mechanical ventilation. This index has been considered valid by some authors, showing that patients with values below $100 \mathrm{rpm} / \mathrm{L}$ achieve independence from ventilatory support ${ }^{18-21}$. This variable is easily measured, does not depend on the patient's cooperation, and is highly predictive of the result of weaning ${ }^{20}$. However, our data show that that variable was not statistically significantly different between the groups. Our case series comprised 7 patients who had no clinical condition for undergoing the test, due to hemodynamic instability or sedation, or both. Similar results were also found by Nava et $\mathrm{al}^{4}$ in patients with COPD; according to those authors, the rapid and superficial respiration index did not prognosticate success or lack of success in weaning.

The $\mathrm{PaO}_{2} / \mathrm{FiO}_{2}$ ratio is routinely used at our institution after cardiac surgery as a parameter of mechanical ventilation weaning, and it may be used independently of the patient's clinical conditions, although cardiovascular alterations may influence venous oxygen content and cardiac output ${ }^{13}$. That ratio identified no significant difference in regard to oxygenation in both groups.

Cardiac dysfunction has been reported in the literature as 1 of the factors that impair mechanical ventilation weaning in patients undergoing cardiac surgery ${ }^{7,8,22}$. Lemaire et $\mathrm{al}^{23}$ reported that COPD and left ventricular dysfunction are factors that may contribute to failure to wean from mechanical ventilation. A statistically significant difference was observed in the incidence of cardiac dysfunction between the groups; the presence of cardiogenic shock and low ventricular ejection fraction were factors that interfered negatively with weaning from mechanical ventilation.

Time of extracorporeal circulation is 1 of the major factors that delay weaning from mechanical ventilation in cardiac surgery, due to the important physiological disorder caused by the inflammatory response to the extracorporeal circuit. This disorder affects the pulmonary capillary membrane, causing an increase in the physiological shunt, and altering gas exchanges ${ }^{24}$. Previous studies have shown that a prolonged extracorporeal circulation time $(>120$ minutes) is usually related to a high surgical risk ${ }^{7,8}$. Our findings were similar in patients who remained in extracorporeal circulation for more than 120 minutes.

The neurological alterations were one of the major complications in the postoperative period of cardiac surgery. The mechanisms involved are multifactorial, although those related to extracorporeal circulation are specifically implicated ${ }^{25}$. Our data show that patients who had undergone cardiac surgery and had neurological alterations, when undergoing prolonged mechanical ventilation did not necessarily evolve to unsuccessful weaning.

In conclusion, our study showed that in patients undergoing cardiac surgery and requiring prolonged mechanical ventilation, data on respiratory system mechanics and oxygenation did not interfere with the success or lack of success in weaning. On the other hand, cardiac dysfunction and time of extracorporeal circulation directly influenced success in weaning.

\section{Acknowledgements}

We thank Dr. Estela Azeka for reviewing the article. 


\section{Referências}

1. Gluck EG, Corgian L. Predicting eventual success or failure to wean in patients receiving long-term mechanical ventilation. Chest 1996; 110: 1018-24.

2. Zanotti E, Rubini F, Lotti G, et al. Elevated static compliance of the total respiratory system: early predictor of weaning unsuccess in severe COPD patients mechanically ventilated. Intensive Care Med 1995; 21 : 400-5.

3. Tobin JM, Laghi F. Monitoring respiratory muscle function. In: Tobin JM. Editor. Principles and Practice of Intensive Care Monitoring. New York: MacGrawHill, 1998; 497-544.

4. Nava S, Rubin F, Zanotti E, et al. Survival and prediction of successful ventilator weaning in CPOD patients requiring mechanical ventilation for more than 21 days. Eur Respir J 1994; 7: 1645-52.

5. Elpern EH, Larson R, Douglas P, Rosen RL, Bone RC. Long-Term outcomes for elderly survivors of prolonged ventilator assistance. Chest 1989; 96: 1120-4.

6. Bernard F, Denault A, Babin D, et al. Diastolic dysfunction is predictive of difficult weaning from cardiopulmonary bypass. Anesth Analg 2001; 92: 291-8.

7. Rady MY, Ryan TA. Perioperative predictors of extubation failure and the effect on clinical outcome after cardiac surgery. Crit Care Med 1999; 27: 340-7.

8. Wong DT, Cheng DCH, Kustra R, et al. Risk factors of delayed extubation, prolonged length of stay in the intensive care unit, and mortality in patients undergoing coronary artery bypass graft with fast-track cardiac anesthesia. Anesthesiology 1999; 91: 911-4.

9. Butler R, Keenan SP, Inman KJ, Siibbald WJ, Block G. Is there a preferred technique for weaning the difficult to wean patient? A systematic review of the literature. Crit Care Med 1999; 27: 2331-6.

10. Carson SS, Bach PB, BrzozowskiI L, Leff A. Outcomes after long-term acute care. An analysis of 133 mechanically ventilated patients. Am J Respir Crit Care Med 1999; 159: 1568-73.

11. Carmona MJC, Auler Jr JOC. Assistência ventilatória no pós-operatório de cirurgia cardíaca. In: Auler Jr JOC, Amaral RVG. Assistência Ventilatória Mecânica. São Paulo: Atheneu, 1995: 285-97.

12. Oh TE, Pierson DJ, Tyler ML. Resistance of humidifiers, and inspiratory work imposed by a ventilator-humidifier circuit. Br J Anaesth 1991; 66: 258-63.
13. Emmerich JC. Monitorização respiratória: fundamentos. Rio de Janeiro: Revinter 1996: 69-109.

14. LoCícero III J, McCann B, Massad M, Joob AJ. Prolonged ventilatory support after open-heart surgery. Crit Care Med 1992; 20: 990-2.

15. Kurek J, Cohen I, Lambrinos J, Minatoya K, Bootf VM, Chalfin DB. Clinical and economical outcome of patients undergoing tracheotomy for prolonged mechanical ventilation in New York state during 1993: analysis of 6.353 cases under diagnosis-related groups 483. Crit Care Med 1997; 25: 983-8.

16. AulerJrJOC, Carmona MJC, Barbas CV, SaldivaPHN, MalbouissonLMS. Theeffects of positive end-expiratory pressure on respiratory system mechanics and hemodynamics in postoperative cardiac surgery patients. Braz J Med Biol Res 2000; 33: 31-42.

17. Van Belle AF, Wesselling GJ, Penn CKM, Wouters EFM. Postoperative pulmonary function abnormalities after coronary artery bypass surgery. Respir Med 1992; 86: 195-9.

18. Tobin JM. Weaning from mechanical ventilation: what have we learned? Respir Care 2000; 45: 418-35.

19. Pierce LNB. Guide to Mechanical Ventilation and Intensive Respiratory Care. Respir. Philadephia: WB Saunders Co., 1995: 34-57.

20. Capdevilla X, Perrigault PF, Ramanataxo M, et al. Changes in breathing pattern and respiratory muscle performance parameters during difficult weaning. Crit Care Med 1998; 26: 79-87.

21. Yang KL, Tobin M. A prospective study of indexes predicting the outcome of trials of weaning from mechanical ventilation. N Engl J Med 1991; 324: 145-50.

22. Hanneman SK. Multidimensional predictors of success or failure with early weaning from mechanical ventilation after cardiac surgery. Nurs Res 1994; 43: 4-10.

23. Lemaire F, Teboul JL, Cinotti L, et al. Acute ventricular dysfunction during unsuccessful weaning from mechanical ventilation. Anesthesiology 1988; 69: 171-9.

24. Westaby S, Parry PA, Reagan DO, et al. Does modern cardiac surgery require conventional intensive care? Eur J Cardiothorac Surg 1993; 7: 313-8.

25. Isgro F, Schmidt, $\mathrm{CH}$, Pohl $\mathrm{P}$, Saggau W. A predictive parameter in patients with brain related complications after cardiac surgery? Eur J Cardiothorac Surg 1999; 116: $640-4$. 